\title{
CONFERENCE MOVED TO 2021
}

CloserStill Media, organiser of the British Dental Conference and Dentistry Show, have taken the decision to postpone the British Dental Conference and Dentistry Show (BDCDS). The 2020 event will now take place on Friday 21 and Saturday 22 May 2021 at the Birmingham NEC, hall 5.

Martin Woodrow, Chief Executive of the British Dental Association (BDA), said: 'It's a real shame that we can't hold what would have been an excellent conference and exhibition this year, but in the circumstances, postponing the 2020 event has to be the right thing to do and I'm sure the 2021 event will be bigger and better than ever!'
In the meantime the event organisers will be focussing on how they can support the profession as they prepare to return to their practice and resume some normality during a very testing time for dentistry.

The BDCDS is the UK's leading two-day exhibition and conference for dental professionals, providing clinical, leadership and practical training via an extensive educational programme and hands-on workshops. It is the UK's largest event of its kind, attracting over 9,000 unique delegates and over 400 exhibitors.

The 2021 event will still be co-located with The Dental Technology Showcase.

\section{Student DCP domates oral hygiene supplies to care homes}

Second year dental hygiene and dental therapy student Tamsyn McKenna from Teesside University has been coordinating the donation of oral care products to care home residents during the Coronavirus pandemic.

Tamsyn approached oral hygiene brands including BioMin, TePe, Piksters and Oralieve to ask if they would be willing to donate their products for the most vulnerable members of society, and received an overwhelming response.

Between them, the companies donated 500 tubes of toothpaste, toothbrushes, dental floss, interdental aids and flossets, which Tamsyn has been busy donating to care homes.

Tamsyn, 23, from Gateshead, said: 'For me, this pandemic has brought to light what is important - helping others. I have managed to complete my university work and still have the time to volunteer and donate to those in need.

I am so grateful to the companies I have been working with for their generous donations. As a student dental hygienist and dental therapist, I understand that good oral health is paramount, and I am pleased to be able to help provide some of the most vulnerable people with access to oral hygiene products.

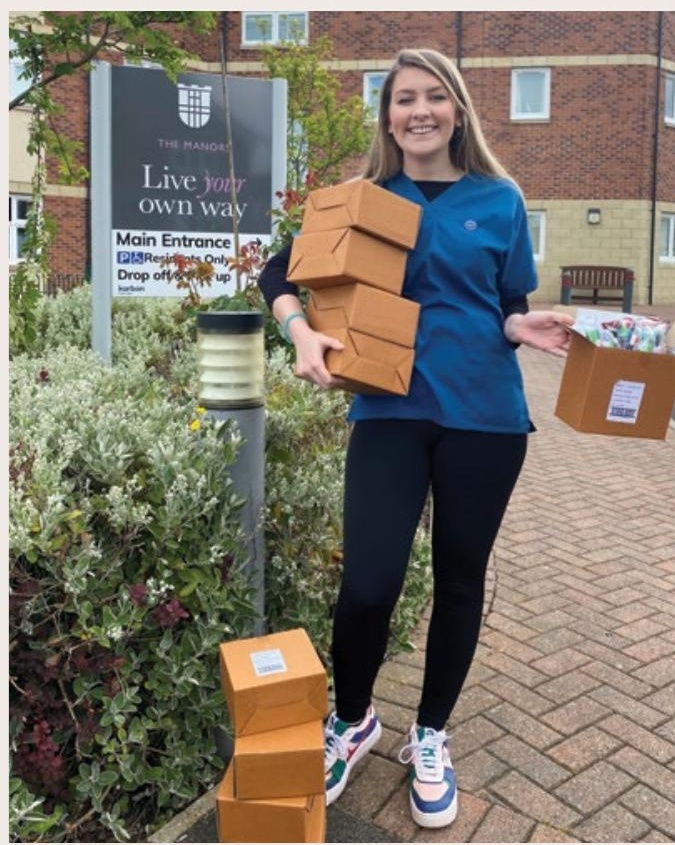

